\title{
Effects of High-Frequency Inflation (HFI) on Expiratory Activity
}

\author{
Masato Sibuya, Arata Kanamaru, Akiyo Isobe, \\ Michiko Iwase and Inuo Homma \\ Department of Physiology, Showa University School of \\ Medicine, Tokyo 142
}

\begin{abstract}
Sibuya, M., Kanamaru, A., Isobe, A., Iwase, M. and Homma, I. Effects of High-Frequency Inflation (HFI) on Expiratory Activity. Tohoku J. Exp. Med., 1988, 156, Suppl., 65-73 — It has been reported that triangular-shaped positive pressure pulses applied to the airway (high-frequency inflation, HFI) stimulates pulmonary stretch receptors and prolongs expiratory duration. In the present experiment, changes in the integrated curve of expiratory activity induced by HFI were investigated. Duration until the onset of the expiratory activity (E1 stage) was unchanged. In the E2 stage, the initial ascending slope became steeper and higher peaks were reached during HFI stimulation. The period from the onset of the slope to the peak, previously designated the stage of active expiration ( $\mathrm{Ea}$ stage), showed no consistent change in duration and remained unchanged on average. The period after the peak until the onset of the following inspiratory activity was consistently prolonged. This period was designated the declining stage of expiration (Ed stage). - expiratory activity; high-frequency inflation; pulmonary stretch receptors; vagal afferents ; expiratory duration
\end{abstract}

Vagal afferent impulses from pulmonary mechanoreceptors are known to modify inspiratory activity. The most classical example is the termination of inspiratory activity by pulmonary stretch receptor (PSR) afferents. This was originally reported by Breuer and Hering, and was later confirmed by others (for review ; Euler 1986). The rate of rise of phrenic activity has also been reported to increase with the increase in lung volume during the inspiratory phase (DiMarco et al. 1981). Irritant receptor afferents are also known to induce augmented breaths (Glogowska et al. 1972). There are several reports indicating that PSR and irritant afferents influence the onset of inspiratory activity and thus have a significant effect on expiratory duration (Stark and Frantz 1979; Rao et al. 1981 ; Homma et al. 1985 ; Trippenbach et al. 1985). On the other hand, less attention has directed toward the effect of vagal afferents on expiratory activity (Bishop 1963 ; Farber 1982).

Pulmonary mechanoreceptors can be stimulated in several ways. Lung inflation, tracheal distension, and positive pressure pulses are known to stimulate PSR, while mechanical irritation (e.g., scratching), lung deflation, negative pressure pulses and pathophysiological changes are known to stimulate irritant recep- 
tors (for review ; Coleridge and Coleridge 1986). Recently, Homma et al. (1987) reported that triangular-shaped positive pressure pulses applied to the airway stimulate PSR. A mode was seen in the cross-correlogram between positive pressure pulses, designated high-frequency inflation (HFI), and vagal afferent impulses from PSR. It was also reported that HFI decreased respiratory frequency by prolongation of the expiratory duration (Homma et al. 1985). However, the effect of HFI on expiratory activity has not been reported. In this study, we investigated the effects of HFI on expiratory activity.

\section{Materials and Methods}

Experiments were performed on 8 rabbits (weighing 2.6 to $3.4 \mathrm{~kg}$ ) anesthetized initially with $450 \mathrm{mg} / \mathrm{kg}$ urethane and $45 \mathrm{mg} / \mathrm{kg}$ chloralose (Wako, Osaka) administered intravenous1y. The trachea was incised about $1 \mathrm{~cm}$ below the upper edge of the trachea and cannulated. The spinous process at Th5-6 and L2-3 levels was explored, pinched and fixed with the rabbit in the prone position, so that the abdomen was lifted from the experimental stage. A pair of wire electrodes were inserted in the abdominal muscle. The position permitted expiratory activity to be recorded even during quiet breathing. The phrenic nerve was detached from the surrounding tissue and cut and the proximal end was desheathed for the inspiratory activity to be recorded from another pair of wire electrodes. The neuromuscular activities were amplified (AB-621G, Nihon Kohden, Tokyo) and recorded on a tape recorder (R-71, TEAC, Tokyo). HFI stimulation was generated as previously reported (Homma et al. 1985). In brief, a moving coil in a sealed box was driven by triangular-shaped electrical pulses. In 4 rabbits, unilateral vagotomy was performed and HFI stimulation was applied during contralateral vagal cooling. Vagal cooling was performed using a thermode with a $1 \mathrm{~cm}$-wide hook-shaped plate at its tip. The thermode has an inner and outer tube, except for $1 \mathrm{~mm}$ at the tip. Pressurized $\mathrm{CO}_{2}$ gas flows distally through the inner tube, expands near the tip, thus cooling the plate, and then flows outward through the outer tube. The temperature of the plate was monitored by means of a thermocouple. The expiratory activity before and during the HFI stimulation were compared by rectifying and integrating the activity.

\section{Results}

Fig. 1 illustrates the effect of $50 \mathrm{~Hz}$ HFI on the expiratory activity. The voltage used to drive the airway pressure pulse generator was changed in 4 steps. HFI increased the expiratory activity. The effect was voltage-dependent, and a larger increase in activity was seen with higher voltage. The effect was transient and the increased expiratory activity promptly returned to the control level after the cessation of the stimulus. As shown in Fig. 2, the expiratory-increasing effect of HFI was also frequency-dependent, as the effect on expiratory duration as reported by Homma et al. (1986). Fig. 2 also shows that HFI can recruit expiratory activity when there is no spontaneous activity in the abdominal muscle. A larger increase was seen with HFI at higher frequencies, such as 50 and $100 \mathrm{~Hz}$. HFI stimulation was applied after unilateral vagotomy and during contralateral vagal cooling (Fig. 3). Spontaneous expiratory activity, and the HFI effect was seen at vagal temperatures as low as $10^{\circ} \mathrm{C}$. At $8^{\circ} \mathrm{C}$, spontaneous expiratory activity disappeared and no activity was recruited. HFI induced no changes in 


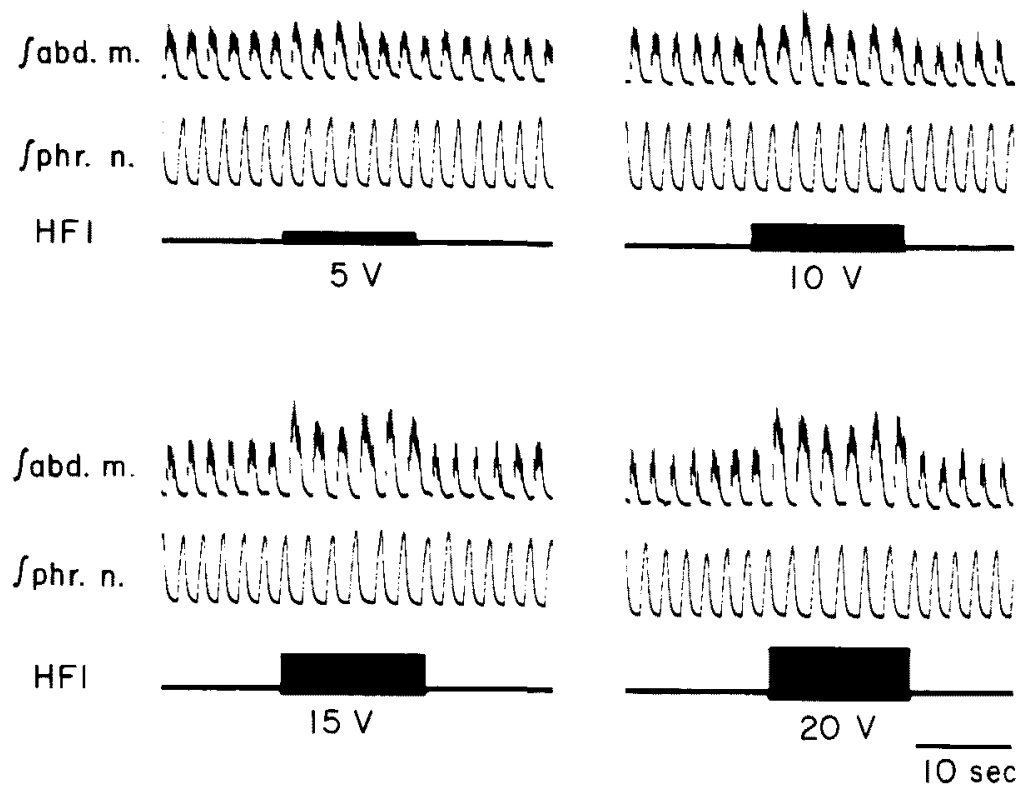

Fig. 1. Effect of $50 \mathrm{~Hz}$ HFI on expiratory activity. Voltage indicated is that used to drive the airway pressure pulse generator. fabd. m., $\int \mathrm{phr} . \mathrm{n}$.; integrated curves of abdominal muscle activity and phrenic nerve activity, respectively.

fabd. m.
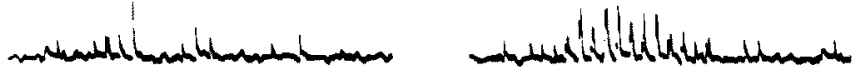

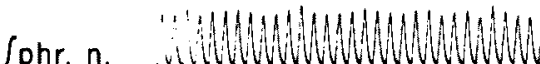

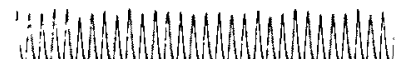

$\mathrm{HFI}$
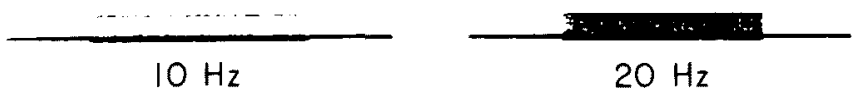

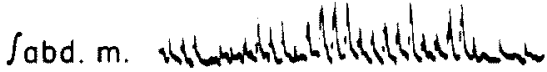



fphr. n
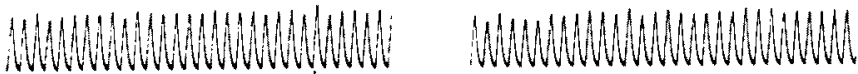

HFI
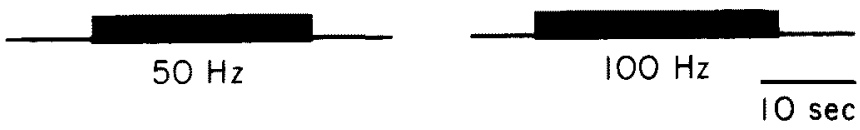

Fig. 2. Frequency property of the expiratory enhancing effect of HFI. Headings are as in Fig. 1. 
$8^{\circ} \mathrm{C}$

Sabd. m.

fphr. n.

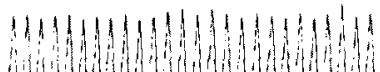

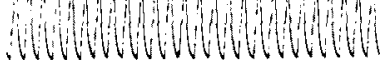

HFI

$15^{\circ} \mathrm{C}$

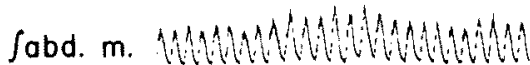

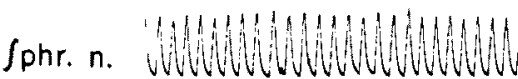

HFI



$10^{\circ} \mathrm{C}$



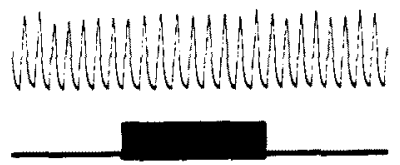

$22^{\circ} \mathrm{C}$

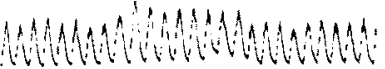



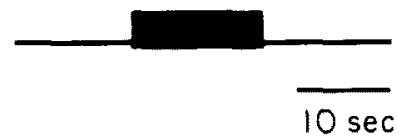

Fig. 3. Changes in the expiratory enhancing effect during vagal cooling. Headings are as in Fig. 1.

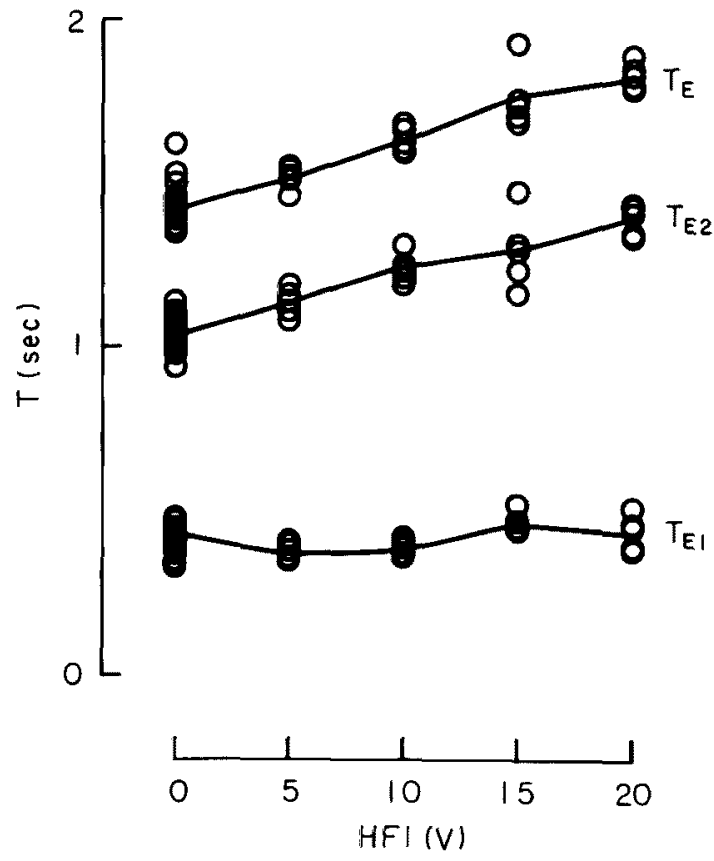

Fig. 4. Changes in the duration of expiration $\left(T_{E}\right)$, first stage of expiration $\left(T_{E 1}\right)$ and second stage of expiration $\left(\mathrm{T}_{\mathrm{E}_{2}}\right)$ induced by HFI. Voltages used to drive the airway pressure pulse generator are shown on abscissa. $O$ indicates the control breaths. 
the inspiratory activity amplitude or duration $\left(\mathrm{T}_{1}\right)$.

The increased expiratory activity was accompanied by prolongation of the expiratory duration $\left(T_{E}\right)$. The voltage-dependency of the $T_{E}$ prolongation is shown in Fig. 4. Zero volt indicates the non-stimulated control breaths. Each plot represents a value determined from one breath. Greater prolongation was seen at higher voltage, as was the increased expiratory activity. The expiratory phase was divided into the first and second (E1 and E2) substages by the onset of the tidal expiratory activity (Richter 1982; Sibuya et al. 1987), and their duration ( $T_{E 1}$ and $T_{E 2}$, respectively) was determined. $T_{E 2}$ was dose dependently prolonged by HFI, while $T_{E 1}$ remained constant. The slope of prolongation of $T_{E}$ and $T_{E 2}$ was identical. This indicates that the prolongation of $T_{E}$ can be explained only by that of $T_{E 2}$. No significant difference was seen between $T_{E 1}$ of the control breaths and that during $20 \mathrm{~V}$ HFI at $50 \mathrm{~Hz}$.

The temporal profile of the enhanced expiratory activity was studied in 12 trials of HFI stimulation in 8 rabbits. In Fig. 5 , integrated expiratory activity of a control breath and a breath, a few seconds later, during $50 \mathrm{~Hz} H F I$ stimulation are superimposed matching the onset of the tidal expiratory activity. A few differences can be noted between the two. The amplitude (height to the peak) of the tidal expiratory activity was increased by HFI, as mentioned above. A significant increase was seen in all 12 trials. The peak was seen before the onset of the next inspiration in both cases. Thus, a substage of the E2 stage during the initial rising slope on the integrated expiratory activity curve (from the onset to the peak) was recently designated by Sibuya et al. (1987) as the stage of active expiration (Ea stage). The rate of rise of the expiratory activity in the Ea stage was increased by HFI in 9 of the 12 trials and remained unchanged in the other 3. However, the duration of the Ea stage $\left(\mathrm{T}_{\mathrm{Ea}}\right)$ remained unchanged. $\mathrm{T}_{\mathrm{Ea}}$ was prolonged in 4, remained unchanged in 4 and was shortened in 4 of the 12 trials. Thus, in general, there were no consistent changes in $\mathrm{T}_{\mathrm{Ea}}$. It can be seen in Fig. 4,



Fig. 5. Effect of HFI on the integrated curve of expiratory activity. Curves prior to (lower) and during (higher) HFI application were superimposed by matching the onset of tidal expiratory activity. 




Fig. 6. Superimposed integration curves of the inspiratory and expiratory activities of the same breath as in Fig. 5, as matched by the onset of the following tidal inspiratory activity. Headings are as in Fig. 1.

that there was more activity after the peak (in the declining phase of the integrated curve) in the breath during HFI stimulation. The plateau continued longer during the stimulated breath and the portion of the E2 stage following the Ea stage was prolonged. Such prolongation was seen in 8 of the 12 trails, while it remained constant in the other 4 . The relation is seen in Fig. 6, in which the inspiratory and expiratory activity of the same breaths as in Fig. 5 were superimposed matching the onset of the following tidal inspiratory activity. The temporal difference in the peak of the expiratory activity indicates the difference in duration of the portion of the E2 stage following the Ea stage. The difference is identical to the difference in $\mathrm{T}_{\mathrm{E}}$ seen in the inspiratory activity. It can be summarized that the prolongation of this period within the E2 stage was responsible for the $T_{E}$ prolongation previously reported (Homma et al. 1985).

\section{Discussion}

In this study, it was shown that, as a result of HFI stimulation applied to the airway, expiratory activity in the abdominal muscle increased. HFI has previously been reported to stimulate PSR (Homma et al. 1986). Thus it may be assumed that PSR afferents increase expiratory activity. There are other reports suggesting that PSR stimulation induces expiratory activity (Bishop 1963; Davies et al. 1980; Farber 1982). This seems logical, as an increase in PSR afferents in a physiological condition indicates inflation of the lungs and an 
increase in expiratory activity would be a reasonable process to reverse that. PSR afferents is also known to terminate inspiratory activity for the same reason. Such an effect was not seen with HFI. HFI was reported to be a specific stimulator of PSR at frequencies over $50 \mathrm{~Hz}$ (Homma et al. 1986). This was consistent with the present finding that HFI at higher frequencies had a greater expiration-increasing effect (Fig. 2). It was also found in the present study that the HFI effect is abolished by cooling the vagal nerve to $8^{\circ} \mathrm{C}$. This is the temperature known to block the conduction of myelinated fibers (Paintal 1965). This indicates that it was the PSR afferents that mediated the expiration increasing effect of HFI.

Sibuya et al. (1987) recently proposed an 'expiratory off-switch' mechanism. According to this theory, expiratory activity continues firing at a certain rate (reflected on the rate of rise of the integration curve) until it reaches the expiratory off-switch level. Then the off-switch mechanism is triggered, expiratory activity suppressed and $\mathrm{T}_{\mathrm{Ea}}$ determined. Applying this theory to the present finding, it can be concluded that PSR afferents both increase the rate of rise and elevate the expiratory off-switch level. These parameters were consistently increased by HFI. However, the change in $\mathrm{T}_{\mathrm{Ea}}$ was not consistent. This indicates that the balance between the increase in the rate of rise and the elevation of the off-switch level was not constant and could vary among rabbits and experimental conditions. This is probably because the two are separately determined by different neural subgroups within the 'expiratory pattern generator'. The anatomical location of these neural networks and their interconnection remain to be determined. The onset of the expiratory activity after termination of inspiratory activity $\left(\mathrm{T}_{\mathrm{E}_{1}}\right.$ ) was also unchanged by HFI stimulation. It has been reported that post-inspiratory inspiratory activity affects the onset of the expiratory activity (Richter 1982).

After the peak, HFI induced lingering expiratory activity before the onset of the next inspiration. This phase is the remainder of the E2 stage following the Ea stage, and is after the peak of the expiratory activity up to the onset of the following inspiratory activity. We designated this period in the E2 stage,the declining stage of expiration (Ed stage). Its duration $\left(\mathrm{T}_{\mathrm{Ed}}\right)$ was prolonged by PSR afferents. This is after the expiratory off-switch mechanism was triggered. Thus, we postulate another independent mechanism responsible for determining the activity and duration of the Ed stage. The most consistent change induced by HFI, concerning duration, was the prolongation of $\mathrm{T}_{\mathrm{Ed}}$. Thus the 'Ed mechanism' has a powerful ability of controlling $T_{E d}$ and also $T_{E 2}, T_{E}$ and the respiratory frequency according to vagal afferents. Various maneuvers that stimulate pulmonary mechanoreceptors are known to modify $T_{E}$. This study indicated the possibility that the expiratory activity is also affected by these maneuvers. It may be of significant importance to divide the expiratory phase into its substages (E1, and E2 further divided into Ea and Ed stages) and identify 
which substage was modified. It is possible that the expiratory off-switch mechanism and/or the Ed mechanism is affected in a certain mode and is the cause of the modified $T_{E}$, as was seen to be the case with HFI.

In summary, PSR afferents induced by HFI increased the expiratory rate of rise and elevated expiratory off-switch level, but did not have any consistent effect on $\mathrm{T}_{\mathrm{E} 1}$ or $\mathrm{T}_{\mathrm{Ea}}$. PSR afferents prolonged $\mathrm{T}_{\mathrm{E} 2}$ and this was due to the prolongation of the portion of the $\mathrm{E} 2$ stage following the Ea stage. This period was designated the declining stage of expiration (Ed stage). It was suggested that the activity and duration of the Ed stage is controlled by an independent mechanism.

\section{References}

1) Bishop, B. (1963) Abdominal muscle and diaphragm activities and cavity pressures in pressure breathing. J. Appl. Physiol., 18, 37-42.

2) Coleridge, H.M. \& Coleridge, J.C.G. (1986) Reflexes evoked from tracheobronchial tree and lungs. In: Control of Breathing, Part 1, edited by N.S. Cherniack \& J.G. Widdicombe, Vol. 2, The Respiratory System, edited by A.P. Fishman, American Physiological Society, Bethesda, Section 3, pp. 395-429.

3) Davies, A., Sant'Ambrogio, F.B. \& Sant'Ambrogio, G. (1980) Control of postural changes of end expiratory volume (FRC) by airways slowly adapting mechanoreceptors. Respir. Physiol., 41, 211-216.

4) DiMarco, A.F., Euler, C. von, Romaniuk, J.R. \& Yamamoto, Y. (1981) Positive feedback facilitation of external intercostal and phrenic inspiratory activity by pulmonary stretch receptors. Acta Physiol. Scand., 113, 375-386.

5) Euler, C. von. (1986) Brain stem mechanisms for generation and control of breathing pattern. In: Control of Breathing, Part 1, edited by N.S. Cherniack \& J.G. Widdicombe, Vol. 2, The Respiratory System, edited by A.P. Fishan, American Physiological Society, Bethesda, pp. 1-67.

6) Farber, J.P. (1982) Pulmonary receptor discharge and expiratory muscle activity. Respir. Physiol., 47, 219-229.

7) Glogowska, M., Richardson, P.S., Widdicombe, J.G. \& Winning, A.J. (1972) The role of the vagus nerves, peripheral chemoreceptors and other afferent pathways in the genesis of augmented breaths in cats and rabbits. Respir. Physiol., 16, 179-196.

8) Homma, I., Onimaru, H., Oouchi, M. \& Ichikawa, S. (1985) The effects of highfrequency inflation and high-frequency deflation on respiration in rabbits. Neurosci. Lett, 60, 307-311.

9) Homma, I., Isobe, A., Onimaru, H. \& Oouchi, M. (1986) Slowly adapting and rapidly adapting pulmonary receptor responses to high-frequency inflation in rabbits. In: Neurobiology of the Control of Breathing, edited by C. von Euler \& H. Lagercrantz, Raven Press, New York, pp. 269-274.

10) Homma, I., Isobe, A., Iwase, M., Onimaru, H. \& Sibuya, M. (1987) Cross-correlation between vagal afferent impulses from pulmonary mechanoreceptors and highfrequency inflation (HFI) and deflation (HFD) in rabbits. Neurosci. Lett., 75, 299302.

11) Paintal, A.S. (1965) Block of conduction in mammalian myelinated nerve fibres by low temperatures. J. Physiol., 180, 1-19.

12) Rao, S.V., Sant'Ambrogio, F.B. \& Sant'Ambrogio, G. (1981) Respiratory reflexes evoked by tracheal distension. J.Appl. Physiol., 50, 421-427.

13) Richter, D.W. (1982) Generation and maintenance of the respiratory rhythm. $J$. Exp. Biol., 100, 93-107.

14) Sibuya, M., Homma I., Hara, T. \& Tsuyama, N. (1987) Expiratory activity in 
transferred intercostal nerves in brachial plexus injury patients. J. Appl. Physiol., 62, $1780-1785$.

15) Stark, A.R. \& Frantz, I.D., III. (1979) Prolonged expiratory duration with elevated lung volume in newborn infants. Pediat. Res., 13, 261-264.

16) Trippenbach, T., Kelly, G. \& Marlot, D. (1985) Effects of tonic vagal input on breathing pattern in newborn rabbits. J. Appl. Physiol., 59, 223-228. 